\title{
Complutum
}

ISSN: 1131-6993

\section{Cuando el futuro corre más deprisa que nosotros. Reflexiones en torno a los diez años de El futuro de la arqueología en España}

Jaime Almansa Sánchez ${ }^{1}$

Recibido: 29/04/2021 / Aceptado: 15/08/2021

Resumen. ¿Hemos llegado ya al futuro? Es la pregunta de partida de una reflexión que busca situar la arqueología española tras diez años de la publicación del libro El futuro de la arqueología en España. A través de un repaso por los diferentes ámbitos que engloban la práctica arqueológica se profundizará en los avances que está viviendo el sector y los retos que siguen pendientes. Un manifiesto por la unión del colectivo desde el análisis crítico de la realidad profesional de la arqueología.

Palabras Clave: arqueología; futuro; profesión; precariedad; esperanza

When the future runs faster than us. Reflections about the ten years of The Future of Archaeology in Spain

Abstract. Did we get to the future yet? This is the starting question for a reflection that aims to draw the situation of Spanish archaeology ten years after the publication of the book, The future of archaeology in Spain. Through a review of the different spheres that shape archaeological practice, this paper will delve into the latest developments in the sector and the pending challenges ahead. A manifest for a unite collective from the critical analysis of the archaeological profession's reality.

Keywords: archaeology; future; profession; precarity; hope

Sumario. De aquellos barros, estos lodos. ¡Ah! Cómo hemos cambiado... La formación. La investigación. La arqueología comercial. La gestión. Los museos y espacios arqueológicos. La divulgación y la transferencia. El colectivo. El futuro de la arqueología en España, versión 2.0. Referencias.

Como citar: Almansa Sánchez, J. (2021): Cuando el futuro corre más deprisa que nosotros. Reflexiones en torno a los diez años de El futuro de la arqueología en España. Complutum, 32(2): 261-273.

«En los pasados años 60 había un coche, el Tiburón Citroën, del que se decía que era como serían los coches del futuro. Después

llegó el futuro y ningún coche fue como aquel» (Criado Boado, 2011: 55).

Hace una década comenzaba una temeraria aventura empresarial en medio de una grave crisis económica que aún no sé cómo pude sortear. Uno de mis primeros proyectos era un libro colaborativo en el que aprovechando la situación nos parásemos a pensar dónde estábamos y qué habíamos hecho para estar así (Almansa Sánchez 2011). Contra todo pronóstico fue un éxito y la gira de presentaciones cual grupo de rock nos llevó a debatir sobre el tema durante más de un año en diferentes foros por toda la península. Hoy han pasado ya diez años desde que esta aventura comenzó. Personalmente he alcanzado algunos objetivos vitales

\footnotetext{
Instituto de Ciencias del Patrimonio, CSIC

jaime.almansa-sanchez@incipit.csic.es
} 
que, aunque tarde, llegaron. La empresa, que gracias a ese libro situó la marca editorial en buena posición, ya no existe. La editorial, sin embargo, no podía desaparecer y se mantiene como si no hubiese pasado nada bajo el paraguas de una nueva asociación en la que lo único que ha cambiado es la forma jurídica. Casi superada la crisis del 2008, un virus ha llevado la economía de nuevo al límite, paralizando el mundo durante meses. Parece que el ciclo vuelve a comenzar ¿Hemos llegado ya al futuro?

\section{De aquellos barros, estos lodos}

Mientras escribo estas líneas, la Society of Antiquaries of London (2020) acaba de publicar un manifiesto por el futuro de la arqueología inglesa en el que se lamenta la descoordinación y aparente declive que vive en la última década. Inmediatamente, las críticas llovieron de algunos de los frentes de la profesión, desmarcándose en las redes sociales de la referencia que el informe hace a sus aportaciones (FAME o CIfA, por mencionar dos de las principales plataformas profesionales del Reino Unido, se quejaban de que sus comentarios no han sido tenidos en cuenta en el informe publicado). Desde el sector se ha sido perfectamente consciente de la situación (por ejemplo, Trow 2016; Wills 2018) y el esfuerzo por mejorarla es constante pese a los envites de las nuevas normas de planeamiento (Belford 2020). En un contexto que siempre se ha mostrado como modelo de éxito más allá de las críticas por su marcada tendencia neoliberal (Zorzin 2015; 2016), empezar a leer reflexiones sobre la raíz de los problemas que sufre el sector - también allí - da a la vez una sensación de esperanza y desasosiego. Esperanza por no vernos tan mal en el contexto general. Al fin y al cabo, los problemas que sufrimos son similares en todo el mundo y era ilusorio pensar lo contrario (ver, por ejemplo, Almansa Sánchez 2020a para el ámbito mediterráneo). Desasosiego porque hasta los modelos más fuertes de nuestro entorno parecen frágiles cuando la política no está de su parte (Novaković et al. 2016). Francia, con su poderosa herramienta de protección pública liderada por el Inrap (Institut national de recherches archéologuiques préventives) y decenas de unidades locales y regionales, teme por la creciente mercantilización del proceso y la entrada de empresas privadas (Demoule 2016: 12; Olivier 2016); En Grecia, con uno de los servicios públicos de patrimonio más potentes e influyentes, la sombra de la precarización y la privatización asoma cada vez más (Plantzos 2018; Poulios y Touloupa 2018; Howery 2020); y así país por país, en una tendencia que marca de algún modo el verdadero interés del patrimonio arqueológico en el contexto de otros problemas sociales. De hecho, entrando ya en España, un pequeño sondeo sobre acceso virtual a ofertas sobre arqueología - realizado durante el primer confinamiento de la covid-19-, ofrece algunas ideas (Almansa Sánchez 2020b), en consonancia con el último sondeo sobre valoración hecho por la Comunidad de Madrid antes de la pandemia (Lobera Serrano 2020): básicamente, más allá de los públicos cautivos que se reproducen en Internet, el desapego de la población (especialmente con menos estudios) por el patrimonio cultural y lo que representa es grande.

Releer El futuro de la arqueología en España hoy puede ser un poco desalentador. Si bien son muchos los aspectos que han mejorado, aún podemos vernos en cada uno de los capítulos. Da la impresión de que los problemas estructurales, sistémicos, sobrepasan a los cambios. ¿Hemos cambiado tanto?

\section{¡Ah! Cómo hemos cambiado...}

En el año 2015 organizamos unas pequeñas jornadas para celebrar el 25 aniversario de la creación de la Sección de Arqueología del CDL de Madrid. Uno de los días, escuchando la intervención de Fernando Velasco (primera Junta Directiva), desmoralizaba ver cómo todos los objetivos profesionales que se habían planteado en 1990 con la creación de la Sección seguían pendientes. Equipo tras equipo se habían dado de bruces con las circunstancias y, aunque el trabajo de cohesión del colectivo estaba dando sus frutos, aún nos veíamos lejos de conseguir nada. Cinco años después de aquel evento puede que estemos a punto de llegar a un punto de inflexión. ¿Estaremos llegando al futuro? Hagamos un repaso de estos diez años.

\section{La formación}

Con la publicación del libro, los primeros grados en arqueología completaban su primer curso. Unos años después, las primeras generaciones de graduados en arqueología de nuestro país salían al mercado laboral en un 
momento en el que se comenzaba tímidamente a remontar el descalabro de la crisis de 2008. Muchos han tenido que emigrar, algunos pudieron quedarse, bien continuando su carrera investigadora, sacando oposiciones o en el sector privado. El panorama no era muy diferente del que se vivió en otros momentos, tal vez con excepción del boom constructivo de los primeros 2000. A pesar de todo, algunos de los aspectos negativos que se pusieron de manifiesto hace diez años (Hernando y Tejerizo 2011) se han acentuado. Las tasas siguen aumentando, los recursos no mejoran, las posibilidades de formación práctica aún son escasas y cierto malestar por la situación entre el estudiantado se mantiene - por ejemplo, viendo los indicadores que publican la Universidad de Granada (2018) o la Universidad Complutense de Madrid (2019), el componente práctico desde la Universidad es el peor valorado con diferencia. No es algo que afecte solo a la arqueología, sino que es estructural a todo el sistema universitario, pero tal vez este caso es más sangrante cuando aún no se han terminado de implantar grados de forma sistemática en otras universidades y se mantiene la dualidad con los grados de Historia o Humanidades y unos másteres que no terminan de encontrar su sitio en el ámbito de la especialización. Además, hay también varias asignaturas pendientes en lo que se refiere a gestión y difusión, que hoy son aspectos esenciales en el futuro profesional de la arqueología.

Por supuesto, los avances son firmes también. Al aumento de los títulos específicos le sigue un mayor número de asignaturas con una carga práctica más importante y temas como el de la arqueología pública van encontrando su sitio (Comendador Rey 2018). La carga vocacional de la arqueología hace que ambas partes se esfuercen más, y eso mejora seguramente la valoración global, aunque no debería ser el único motor.

Puede que el aspecto más problemático tenga que ver con la precarización creciente del mundo universitario y la necesaria renovación generacional que se avecina - no por querer cambiar docentes, sino porque la espina dorsal del sistema universitario español se jubila sin una solución clara de continuidad. Las plazas salen con cuentagotas, las condiciones del profesorado asociado son precarias y controvertidas (Díaz Santiago 2013) y las últimas propuestas de carrera profesional hacen a las nuevas generaciones echarse a temblar
(CCOO 2021). La excelencia que se pretende en el ámbito investigador/universitario no es compatible con las condiciones laborales que se ofrecen y la precariedad generalizada del sector. Tal vez este sea uno de los puntos más problemáticos de la realidad actual y nos lleva directamente al siguiente punto.

\section{La investigación}

Creo que es esencial resaltar el salto cualitativo que se ha dado en la última década con respecto a este tema. Sin dejar de lado los problemas a los que volveré después, la presencia de la arqueología española a nivel internacional es mucho más potente.

Podríamos plantear que corresponde a un salto generacional ligado a la mayor internacionalización de las nuevas generaciones, pero creo que se trata de una madurez internacional generalizada. Me explico. Un vistazo a las listas del SJR (Scimago Journal and Country Rank) nos muestra cómo en los últimos años nos hemos consolidado en el top 5 global por citas $\mathrm{y}$ hemos aumentado el número de revistas indexadas considerablemente (de 4 en 2010, ninguna de ellas en el primer cuartil, a 20 en 2019, con dos en el primer cuartil). Números que podemos contrastar con el análisis bibliométrico de Xose-Lois Armada (2016) en el dossier de la Revista d'Arqueologia de Ponent al respecto (Bouso, Rafel y Alonso 2016), en su comparación con el hecho apenas siete años antes (Armada 2009). Parece claro de todos modos que los editores $-\mathrm{y}$ aunque yo no participé, en esto me incluyo- siguen poniendo por delante la defensa de sus líneas editoriales y de la distribución de contenidos de calidad a los modelos neoliberales de excelencia que priman hoy (la literatura al respecto es demasiado extensa y afecta a todas las áreas de conocimiento en un movimiento que se ve agravado por los costes del acceso abierto). Pero como plantea el profesor Ruiz Zapatero en el mismo dossier, «la inclusión de buenos artículos en inglés en nuestras revistas creo que es una de las pocas buenas alternativas para incrementar su prestigio - aunque no todos lo consideran así- y luchar por nuestro posicionamiento internacional» (Ruiz Zapatero 2016: 276). Situación que se está produciendo cada vez más y para la que el modelo de acceso abierto de nuestras revistas puede ser determinante. En este sentido, España se está colocando como uno de los garantes del 
acceso abierto, con gran cantidad de revistas en acceso abierto real ( $\sin$ coste para los autores) y el desarrollo de repositorios públicos para datos en la mayoría de las instituciones. Solo nos falta seguir usando estos recursos y potenciarlos con políticas de investigación que primen esto en lugar de una cuestionable excelencia. Puede que la adhesión de la Agencia Estatal de Investigación a la «Declaración de San Francisco sobre la Evaluación de la Investigación (DORA)» en marzo de 2021 mejore este panorama en un futuro próximo.

Dentro de la tendencia pesimista en la que vivimos y de los problemas de financiación y estabilización que seguimos arrastrando, los resultados que están sacándose adelante son muy positivos. La entrada en escena, por ejemplo, de la Fundación Palarq, ha permitido potenciar aún más la presencia de la arqueología española en el exterior, aunque a la vez ponía de manifiesto la insuficiencia de las opciones existentes tras el declive que trajo la crisis a las convocatorias del Ministerio de Cultura.

A pesar de ello, la investigación dentro de nuestras fronteras tampoco goza de una salud económica excelente. Muchas autonomías han reducido considerablemente sus presupuestos y se siguen financiando proyectos a una fracción de su coste. Por desgracia apenas contamos con revisiones de este tema (ver el caso de Cataluña en Junyent y López 2019) y en el Estado de las Autonomías la variabilidad es demasiado amplia para poder llegar a conclusiones sin analizar los datos.

Algunas críticas feroces como la de González Ruibal (2011) trajeron polémica en su momento, pero no han dejado de estar presentes en el panorama actual como aspectos estructurales de la Academia. Mientras escribo estas líneas, el Ministro de Ciencia e Innovación presenta el proyecto de reforma de Ley de la Ciencia, sobre el papel aparentemente innovador, pero muy protestado por la mayor precarización que parece traer (refrendada por el informe del Ministerio de Trabajo). La generación postdoctoral sigue sin tener reconocidos derechos laborales básicos que se están ganando en los tribunales, como los trienios y sexenios (CCOO $[\mathrm{P}]$ 2021), y el panorama futuro no tiene vistas a mejorar, al menos sin lucha.

\section{La arqueología comercial}

Otro sector bastante precarizado es el de la arqueología comercial. El impacto de la crisis ha sido importante $\mathrm{y}$, lejos de una reestructuración necesaria del ecosistema empresarial, parece que estamos reproduciendo el modelo dentro de una aparente nueva estabilidad en la que sobreviven algunos viejos actores y pocas nuevas iniciativas (Almansa Sánchez 2014).

Los informes de DISCO (Discovering the Archaeologists of Europe) nos aportaron una radiografía muy interesante del panorama, inmediatamente antes de la crisis (Aitchison 2009) y en su fondo (Aitchison et al. 2014). La destrucción de empleo ha sido evidente, el cierre de empresas también, pero el principal problema para España seguía estando en el modelo (Parga-Dans, Barreiro y Varela-Pousa 2016; Morín de Pablos y Barroso Cabrera 2014). La crisis no trajo una transformación en el modelo de trabajo, lastrado por un principio básico: la empresa de arqueología tiene que servir a dos amos, la promotora y la administración (Almansa Sánchez 2017a; López Martínez y Martín Alonso 2018). Esta situación hace que la competencia principal sea la económica, dentro de un sector desregulado en el que el parón en la lucha colectiva ha seguido favoreciendo unas condiciones de precariedad con una diferencia. Si hasta la crisis el peso de esta precariedad caía principalmente sobre los salarios de las personas contratadas, ahora la precariedad se da en forma de autoexplotación. La configuración del sector lo hace así, con el crecimiento de microempresas y fuerza de trabajo autónoma (aún no bien mapeada en nuestro país). Una realidad que se vive también en campos emergentes como el de la Arqueología Virtual (Aparicio 2018).

El hartazgo es patente en el colectivo (sobre lo que volveré más adelante), pero seguimos sin plantearnos una meta realista o una solución al «mientras tanto». En los últimos años se han alzado algunas voces con propuestas variadas - sobre el papel creo que solo la de Rodríguez Temiño y Afonso Marrero (2019), que plantean la creación de un cuerpo de Arqueólogos Profesionales-, pero estemos de acuerdo o no con estas y otras soluciones, lo que está claro es que el inmovilismo no va a solucionar las cosas. Al menos, la crisis actual de la covid-19 ha mostrado cierto avance en la reacción con informes como el que se hizo de impacto económico en el sector ante el primer confinamiento (Colegio de Profesionales de la Arqueología 2020), y parece que vuelve a haber ruido sindical con la creación reciente de las coordinadoras de arqueología de la CNT en Valencia y Madrid. 


\section{La gestión}

Claramente, todo está ligado a los modelos de gestión vigentes, que de algún modo marcan la práctica diaria en todos los sentidos. A pesar de que se ha intentado implantar una forma de arqueología preventiva basada en el principio del que contamina paga (polluter pays principle), los intentos originales de establecer modelos propios se vieron diluidos en un proceso burocratizado de planeamiento (para Madrid, ver Castillo 2004) que se desarrolló con sus pequeñas especificidades en cada una de las Comunidades Autónomas. El principio se mantuvo, la prevención, dependiendo del proceso y el lugar.

En todo caso, en el ámbito de la gestión existen dos aspectos que me parecen graves aun hoy: primero, la arbitrariedad generalizada desde las administraciones (haya o no reglamento), que genera bastantes conflictos internos en el colectivo. Segundo, la amenaza de flexibilización de la protección que vino con la cuarta ola de leyes, en suspenso desde la sentencia del Tribunal Constitucional (122/2014, de 17 de julio) que anuló parcialmente la nueva ley madrileña.

Sobre el primero, se trata de algo que he podido documentar en muchos países del ámbito mediterráneo con mi proyecto actual (aún sin publicar, pero basado en entrevistas con dos centenares de profesionales), incluida España. Sigue siendo una queja «de barra de bar», pero el colectivo comienza a movilizarse con requerimientos desde los Colegios profesionales para aclarar ciertas dinámicas y protestar por el desamparo.

Sobre el segundo, la profesora Querol (2010: 46) planteaba el surgimiento de una cuarta generación de leyes que comenzaría a sustituir o enmendar a las de primera o segunda generación. Una generación que surge a mediados de la primera década del siglo, pero sufrirá un vuelco inesperado con la aprobación sin consenso en 2013 de sendas nuevas leyes en Madrid y Castilla la Mancha (seguidas en 2016 de la nueva ley gallega). Las dos últimas (Ley 4/2013, de 16 de mayo, de Patrimonio Cultural de Castilla la Mancha y Ley 5/2016, de 4 de mayo, del Patrimonio Cultural de Galicia) pasaron con críticas y pequeñas modificaciones ante las enmiendas presentadas (Benítez de Lugo 2013; Barreiro y Varela-Pousa 2017), pero la primera (Ley 3/2013, de 18 de junio, de Patrimonio Histórico de la Comuni- dad de Madrid) salió adelante con la oposición de todo el colectivo y el espectro político del momento en un bronco proceso que terminó en recurso al Tribunal Constitucional y con la ley parcialmente anulada un año después (García Fernández 2014; Díaz, Torija y Zarco 2014; Almansa Sánchez 2017b).

Desde entonces el panorama parecía estar tranquilo. El interminable ciclo electoral que vivimos en los últimos años ha paralizado la actividad legisladora en patrimonio a pesar de que nunca ha dejado de haber rumores de nuevas leyes, incluso una nueva ley nacional que coletea desde 2003 (Almansa Sánchez 2017a: 193-194) y que se reactivó y abortó en pocos meses con un anteproyecto de reforma que trata de dar mayor seguridad jurídica a algunos aspectos que afectan a las competencias ministeriales, reforzando los mínimos de protección $\mathrm{y}$ anunciando una nueva ley para 2023. No cabe duda de que la acción legislativa continuará, aunque es difícil aventurar el cómo en el contexto político actual.

\section{Los museos y espacios arqueológicos}

Puede que este sea uno de los campos en los que más movimiento ha habido. A la publicación del libro se acababan de inaugurar el Museo de la Evolución Humana en Burgos, el Museo Nacional de Arqueología Subacuática en Cartagena, y algunas nuevas sedes de museos provinciales y locales por toda la península como el Museo de Medina Azahara. Algunas reformas en curso como la del Museo Arqueológico de Córdoba, el Museo d'Arqueologia de Catalunya y el Museo Arqueológico de Asturias verían la luz en esos meses. Desde entonces no ha cesado la lista, desde la reapertura del Museo Arqueológico Nacional tras su remodelación en 2014 y seguido del Museo de Málaga en 2016, el Ibero de Jaén en 2017, o la reapertura del Museo de Granada en 2018. Además, se anunciaron las nuevas sedes del Museo Arqueológico de Cantabria y el Museo Nacional de Arte Romano de Mérida. Si nos pusiésemos a hablar de espacios arqueológicos, desde la inauguración del Antiquarium de Sevilla en 2011 la lista se nos haría demasiado larga.

Ahora bien, a la alegría que nos dan estas (re)aperturas, se han sumado multitud de problemas en muchos de estos espacios, desde su propia gestión a la sostenibilidad de su apertura (fue sonado el problema con los parques ar- 
queológicos de Castilla la Mancha, poco tratado en la literatura académica [Cebrián 2016]). Se siguen construyendo infraestructuras sin una visión de futuro clara (ver por ejemplo el conflicto sobre las colecciones para el Museo Ibero de Jaén), aunque también han emergido con fuerza espacios sostenibles y muy activos donde la relación con la comunidad es muy estrecha. Por lo general esta relación se construye desde la iniciativa privada (cooperativas, asociaciones y alguna empresa), y es seguramente una de las pocas consecuencias positivas de la crisis, pero también hay casos de colaboración como el de la Colección Museográfica de Gilena (ver Ruiz García 2020), o el MASAV, Museo Abierto de las Sierras de Ávila y el Valle de Amblés (ver López García 2019), que son dignos de mención.

Desde que se puso en marcha el Laboratorio Permanente de Públicos de Museos para los museos estatales en 2008, contamos con más herramientas de evaluación (ver una revisión general en Pérez Santos 2020), aunque los informes siguen apareciendo con cuentagotas $y$ en otros museos fuera de esta red la situación necesita mejorar con urgencia. La museografía de nuevos espacios sigue siendo muy conservadora, salvo en algunas exposiciones temporales o espacios privamos como CaixaForum, y la calidad de las musealizaciones de espacios arqueológicos llevaría a un amplio debate. Frente a las grandes infraestructuras para enmarcar los yacimientos, los contenidos interpretativos no suelen estar a la altura a pesar de los grandes esfuerzos que hacen algunos espacios y los avances en interpretación y evaluación que se alcanzan día a día.

Tal vez, uno de los marcadores más positivos puede ser el que ofrece la encuesta de hábitos y prácticas culturales del Ministerio de Cultura. Si comparamos los datos de 2010-2011 (MCU 2011) con los de 2018-2019 (MCU 2019), el aumento del interés público por el patrimonio cultural y la arqueología parece haber aumentado. La visita a monumentos y museos sube 10 puntos $\mathrm{y}$, en el caso de los yacimientos arqueológicos tenemos un aumento algo menor pero igualmente significativo: del $29,3 \%$ de interés y el $13,9 \%$ de visitas en el último año, se pasa a un $36,9 \%$ de interés y un $21,8 \%$ de visitas en el último año. Puede que los avances en divulgación y transferencia hayan tenido algo que ver con esto, unidos al mayor dinamismo generalizado de la disciplina en los últimos años.

\section{La divulgación y la transferencia}

No cabe duda de que el papel de la divulgación y el crecimiento y valor de la arqueología pública en nuestro país ha sido mayúsculo. Sobre el panorama general que podríamos atisbar a principios de la década (e.g. Almansa Sánchez 2013), se ha seguido construyendo una bibliografía importante en torno a los diferentes temas que engloban la práctica, con un foco especial en la divulgación y la socialización del patrimonio arqueológico. Si tuviese que destacar un proyecto, sería el impulsado desde el Instituto Milá i Fontanals del CSIC en los últimos años (Gibaja et al. 2021), por su impacto, sostenibilidad y frescura. Pero no es para nada el único y otras iniciativas por todo el territorio nacional darían para una lista importante proyectos participativos y divulgativos relacionados con la arqueología y al margen de los espacios tradicionales. Incluso se ha visto un cambio radical en el ámbito universitario con la aprobación del sexenio de transferencia desde la ANECA, que comenzó a evaluarse de forma piloto en 2019 (aunque con unos requisitos un tanto extraños, que seguían premiando la transferencia comercial e industrial). Cosas que no contaban, ahora comienzan a contar más.

En este sentido, uno de los avances más importantes ha sido el que han traído las redes sociales y otros medios alternativos de comunicación como podcast, vlogs, etc. Con un uso incipiente en 2010, la pandemia de la covid-19 ha hecho de las diferentes redes disponibles una herramienta fundamental para mantener la actividad cultural en tiempos de restricciones y confinamiento (ver González Sánchez 2021), convirtiéndose también en una herramienta esencial para el turismo arqueológico (Corpas y Castillo 2019). A pesar de ello, aún estamos bastante limitados de cara al alcance, manteniendo por lo general los mismos círculos que tenemos en persona y con unas tasas de participación bajas que apenas rompen las pocas cuentas «influencer» que tenemos a día de hoy — una tendencia que pude comprobar en el citado estudio llevado a cabo durante el primer confinamiento en 2020 (ver Almansa Sánchez 2020b) y con el análisis de las redes sociales de los Congresos de Buenas Prácticas en Patrimonio Mundial (Castillo Mena y Almansa Sánchez 2021).

La tecnología es, sin lugar a duda, el principal aliado que estamos teniendo en este ámbi- 
to. Cada año surgen nuevas herramientas y se democratizan otras. Un caso excepcional es el crecimiento que está teniendo el ámbito de las reconstrucciones virtuales, tanto en cantidad como en calidad, y sin descuidar el rigor (ver Aparicio y Figueiredo 2016). En esta línea, el conflicto con la ilustración clásica parece no existir y todo el colectivo se ha embarcado en ADARQ (Asociación Nacional de Dibujantes e Ilustradores de Arqueología), nacida en 2015 y uno de los grandes ejemplos del avance positivo que parece que estamos viviendo en el colectivo.

En el campo de la prensa, agencias especializadas en ciencia como Materia, que ahora trabaja para El País y abrió con dos noticias relacionadas con la arqueología en julio de 2012, o el crecimiento constante de The Conversation, una plataforma que nos acerca de primera mano a la comunicación científica (y también arqueológica), han ayudado a mejorar la calidad de los contenidos, aunque los titulares desafortunados no cesan, especialmente en medios locales. La década comenzó con el estreno en 2011 de Arqueomanía en La 2 de RTVE y acaba de estrenarse (2021) El condensador de flu$z o$ en la misma cadena con un éxito abrumador. Aunque la pseudoarqueología sigue gozando de buena salud en la televisión española, parece haber una contramedida de éxito en la que la profesión se ha implicado activamente.

Ahora que la divulgación y la comunicación parecen estar asentándose, parece el momento de consolidar también una transferencia de conocimiento más profunda y dinámica que pueda tener un impacto real en nuestro futuro como sociedad (Almansa Sánchez 2018).

\section{El colectivo}

Este ha sido uno de mis campos de batalla más encarnecida en la última década. Como en una montaña rusa, el ritmo frenético y los buenos resultados de muchas actividades de las que organizamos desde Asociación Madrileña de Trabajadores y Trabajadoras de la Arqueología (AMTTA), la Sección de Arqueología del CDL de Madrid y otros colectivos, se truncaba con la frustración de no ver un avance claro en ninguna de las reivindicaciones clásicas que estaban sobre la palestra desde finales de los años 80 (Querol y Martínez Navarrete 1994; Martínez y Querol 1996: 57-8). La jornada a la que hacía referencia al comienzo de este apartado fue crucial en ese sentido.
Sin embargo, las últimas semanas nos han traído un nuevo empuje esperanzador en la creación de la Plataforma Estatal de Profesionales de la Arqueología (PEPA) tras un proyecto liderado por varias secciones colegiales en los últimos dos años. Tal vez la semilla sembrada va por fin dando sus frutos. Ahora bien, seguimos teniendo mucho camino por delante para afianzar una posición aún débil.

En este sentido, un aspecto que creo que sí ha cambiado desde la publicación del libro es una mayor conciencia colectiva dentro de la arqueología. Los conflictos internos continúan, seguimos adoleciendo de muchos de los problemas que se exponían hace una década, pero la masa crítica de profesionales dispuestos a superar estas barreras ha crecido lo suficiente como para que las acciones tengan una repercusión más estable. Y no es un proceso sencillo. La mediación se está comenzando a explorar como herramienta en el contexto del patrimonio arqueológico (e.g. Corpas 2020, para el caso de expolio y tráfico ilícito), pero lo que ha sido chocante es que ha sido también necesaria en el proceso de constitución de la PEPA y las acciones concretas por ahora se están limitando a una protección básica de la profesión que debía venir por defecto desde hace años. Se ha comenzado a ganar visibilidad, pero aún no se ha ganado fuerza.

Un aspecto que me parece esencial traer a colación ahora es el avance importante en la visibilización de dos cuestiones fundamentales: género y salud. Esto ha llevado aparejados aspectos muy transversales a toda la profesión y en los que la arqueología española está jugando un papel fundamental, también a nivel internacional. No solo han surgido con fuerza nuevas propuestas y proyectos de visibilización de las mujeres en arqueología (como las actividades y «editatonas» de Wikipedia del grupo de arqueólogas feministas del CDL de Madrid, o el recién comenzado proyecto arqueólogAs en la Universitat de Barcelona), sino también otros trabajos críticos (e.g. Zarzuela, Martín y Donat 2019), que miran más allá de los dualismos tradicionales desde una perspectiva queer (e.g. Moral 2016), o afrontan por fin el problema del acoso en arqueología, con iniciativas como el protocolo de Arkeogazte (Comisión Feminista Arkeogazte 2018) o el informe resultado de la acción emprendida durante el encuentro de la European Association of Archaeologists (EAA) en Barcelona en 2018 (Coto-Sarmiento et al. 2020). Todo esto se ha enmarcado en una 
visión más «sensible» de la profesión, que nos lleva a plantearnos problemas de salud física y mental por primera vez (e.g. Aranburu-Mendizabal et al. 2019; Almansa Sánchez y Díaz de Liaño 2019; Canosa Betés y Díaz de Liaño 2020; Díaz de Liaño, Corpas y Moral 2020).

Desgraciadamente es imposible abordar en un artículo de estas características todos los temas que salen al paso en su justa medida, pero este pequeño ojeo a los principales temas que se abordaron originalmente en el libro nos ayuda a hacernos una idea de cómo, aunque en ocasiones parece que no nos movemos demasiado, sí que se producen novedades importantes en nuestra profesión que ayudan a ver el futuro con otros ojos. Al menos tantas como los inmovilismos casi estructurales que nos mantienen atados a los males de nuestro colectivo. Entonces, ¿hemos llegado ya al futuro?

\section{El futuro de la arqueología en España, versión 2.0}

Algo que me resultó curioso en su momento es que parece que no sabemos mirar al futuro. Cuando pido este ejercicio, terminamos mirando atrás o, como mucho, a un futuro inmediato que se difumina con el presente. Bromeaba al respecto con la propia idea de arqueología. $\mathrm{Si}$ nos pasamos el día tratando de construir mejor el pasado, cómo nos íbamos a plantear construir un futuro. Pero como reza el dicho: «entre broma y broma, la verdad asoma», y como me comentaba hace un par de años Ignacio Rodríguez Temiño en una de nuestras interminables conversaciones al respecto, parece que lo que nos pasa, es que no sabemos a dónde queremos llegar (casi parafraseando a Ortega y Gasset). Hemos sido capaces de definir nuestros males, pero no de mirar al frente y sobreponernos. Si acaso, muchos de los avances son empujones ante una realidad que apremia.

Imaginar cómo estaremos dentro de diez años es difícil, no porque el Tiburón no se vaya a parecer a los coches del futuro, sino porque muchas veces no tenemos una idea clara de qué tipo de coche querremos o podremos tener. La incertidumbre y el cortoplacismo en que vivimos - en la mayoría de las ocasiones marcados por un contexto ajeno a la profesiónnos mantienen en una vorágine de constante preocupación por un mañana demasiado literal. Eventos como el de la covid-19 nos han enseñado que cualquier planificación se puede truncar de un día para otro sin que podamos hacer nada por evitarlo. A pesar de todo, no se puede dejar de lado una perspectiva más amplia ni creo que lo estemos haciendo.

La arqueología tiene futuro porque tiene una capacidad de reinventarse como pocas disciplinas. Si nos fijamos en mi (pre)ocupación principal, la arqueología pública, el cambio está siendo sustancial, desde la práctica irrelevancia a un elemento pivotante del presente y el futuro de nuestra actividad. No solo en aspectos ya comentados, sino en el camino a una mayor relevancia social o, al menos, presencia pública. Este de hecho, sería uno de los caminos que veo más claramente abiertos, tanto en el ámbito de la investigación como en el de la profesión liberal. Tal vez estemos aún lejos de ser intelectuales públicos (Tarlow y Nilsson Stutz 2013), pero nuestra presencia mediática es cada vez mayor, tanto en lo que se refiere a la presentación de nuevos hallazgos y actividades, como en relación con la repercusión de nuestro trabajo en el presente. Tal vez lo que debemos cuidar es que todo esto se sitúe en un contexto positivo tanto para la profesión, como para la sociedad.

En esta línea, no son pocos los trabajos que traen en los últimos años la idea de bienestar en relación con la arqueología (para el último volumen, ver Watson 2021). Es una idea de fondo que lleva tiempo gestándose, especialmente en el ámbito anglosajón, con experiencias que giran entre la arqueología comunitaria (Sayer 2015), la terapia de veteranos (Winterton 2014; Everill, Bennet y Burnell 2020) y la propia inclusión de colectivos con discapacidad (O’Mahony 2015; Farrell-Banks 2018). No me cabe duda de que la semilla está también sembrada en nuestro país y veremos iniciativas interesantes en este sentido.

Con respecto a la arqueología pública, ya apunté en otro lugar la necesidad de explorar los límites de la disciplina (Almansa Sánchez 2018), y considero que es algo sano para la arqueología como tal. De hecho, es algo que se hace constantemente casi sin darnos cuenta, desde la aplicación de tecnologías al desarrollo de nuevas aproximaciones teóricas. En este sentido, la arqueología contemporánea va a tener un papel relevante a la hora de explorar metodologías y aplicar una verdadera transdisciplinariedad. Ya tenemos una presencia destacada en esta línea, con investigadores de referencia internacional como Alfredo González Ruibal, desde su artículo hace más de una déca- 
da en Current Anthropology (González Ruibal 2008) al último libro en Routledge (González Ruibal 2018). Todo ello en un panorama investigador creciente donde de nuevo se cruzan el ámbito investigador y comercial. Pero también ejemplos de investigación transdisciplinar que pueden abrir nuevas líneas de trabajo como la arqueometría forense (Rodríguez Temiño et al. 2018) o la aproximación al espacio desde la cognición (Criado Boado et al. 2019).

Como apuntaba más arriba, el acceso abierto es una de las líneas básicas de cara al futuro de la ciencia y de nuestra profesión. Como parte de él, el llamado open data (datos en abierto), va a ser fundamental. En investigación cada vez está más patente, especialmente en relación con proyectos europeos, pero en el ámbito de la arqueología comercial sigue siendo una asignatura pendiente. A pesar de las leyes de transparencia y de la aparente apuesta por fomentar los repositorios públicos, el acceso a los datos sigue siendo farragoso y poco uniforme (cuando no directamente imposible). Va a ser esencial potenciar fuera del ámbito académico este aspecto $\mathrm{y}$, teniendo en cuenta las diferentes velocidades de las regiones y que actualmente no hay ningún socio desde las administraciones en proyectos como Ariadne+ (al contrario, por ejemplo, que Portugal), puede que en el futuro más inmediato esto siga siendo una asignatura pendiente.

Resulta desalentador, ya que podría facilitar trabajos de síntesis (en el sentido de, Altschul et al. 2019) que nos ayudaran a avanzar en nuevas formas de entender el pasado y el presente, por no hablar de las tan añoradas revisiones de los conjuntos excavados en las últimas décadas. Esto supondría un salto cualitativo de cara a la investigación tradicional (no se entienda de forma peyorativa, pues la considero esencial para continuar avanzando), abriendo nuevas preguntas de investigación, cuestionando hipótesis actuales y resolviendo algunas cuestiones pendientes.

Pero puede que el área en el que más avances veamos sea el del patrimonio. Aún minoritario, su carácter interdisciplinar le da un potencial innovador interesante, especialmente con un cambio de los modelos de gestión (ver por ejemplo el reciente monográfico de la revista PH [Burgos Barrantes 2020]). La arqueología puede tener un papel mayor y las repercusiones serían con seguridad positivas. Será esencial que todo el colectivo se conciencie de esto. Nos hemos fijado mucho en la participación social, pero el público «arqueológico» sigue descuidado y en un momento en el que prima la arqueometría sobre todo lo demás, no podemos olvidar que sin cohesión en el colectivo no podremos conseguir cambios efectivos y generalizados, incluso si esos cambios tienen que subirse al carro del decrecimiento (Flexner 2020, Zorzin 2021) en un momento en el que todo parece insostenible.

Dentro de diez años volveré a mirar atrás y espero que al releer este texto no sienta desasosiego. El futuro llega irremediablemente y con él cambios, más o menos sutiles. Diez años no son mucho tiempo y, aún así, hemos visto un salto cualitativo en la profesión que llama a ser optimistas. Si en la courte durée ya podemos apreciar tantos cambios, ¿qué no vamos a esperar del futuro?

\section{Referencias}

Aitchison, K. (2009): Discovering the Archaeologists of Europe. Transnational report. Institute for Archaeologists, Reading.

Aitchison, K. et al. (+57) (2014): Discovering the Archaeologists of Europe 2012-14: Transnational report. York Archaeological Trust, York.

Almansa Sánchez, J. (ed.) (2011): El futuro de la arqueología en España. JAS Arqueología Editorial, Madrid.

Almansa Sánchez, J. (ed.) (2013): Arqueología Pública en España. JAS Arqueología Editorial, Madrid.

Almansa Sánchez, J. (2014): Bendita crisis, maldita profesión. Arqueoweb, 15: 322-325.

Almansa Sánchez, J. (2017a): Arqueología y Sociedad. Interacción y Acción desde la Teoría Crítica. Universidad Complutense de Madrid. Tesis Doctoral.

Almansa Sánchez, J. (2017b): Power, Politics, Laws and the Management of Archaeological Heritage. An experience from Madrid. Archäologische Informationen, 40: 59-66

Almansa Sánchez, J. (2018): New paths for the future of public archaeology? Cuadernos de Prehistoria $y$ Arqueología de la Universidad de Granada, 28: 197-209. DOI: https://doi.org/10.30827/cpag.v28i0.8482 
Almansa Sánchez, J. (2020a): Spaces for Creativity in Mediterranean Archaeological Heritage Management. Advances in Archaeological Practice, 8(3): 275-287. DOI: https://doi.org/10.1017/aap.2020.21

Almansa Sánchez, J. (2020b): Visitas virtuales durante el confinamiento de la Covid-19. Digital CSIC. DOI: http://dx.doi.org/10.20350/digitalCSIC/12684

Almansa Sánchez, J. y Díaz de Liaño, G. (2019): Sufriendo para ser arqueólogos... Sobre salud y práctica arqueológica. Revista Arkeogazte, 9: 159-174.

Altschul, J.H., Kintigh, K.W., Klein, T.H., Doelle, W.H., Hays-Gilpin, A., Herr, S.A., Kohler, T.A., Mills, B.J., Montgomery, L.M., Nelson, M.C., Ortman, S.G., Parker, J.N., Peeples, M.A. y Sabloff, J.A. (2019): Fostering Collaborative Synthetic Research in Archaeology. Advances in Archaeological Practice, 6(1): 19-29. DOI: https://doi.org/10.1017/aap.2017.31

Aparicio, P. (ed.) (2016): Archaeology and Neoliberalism. JAS Arqueología Editorial, Madrid.

Aparicio, P. (2018): Mercenarios es la selva del precariado: construyendo la arqueología virtual como profesión. Congreso Nacional de Arqueología Profesional (Zaragoza 2017). En Lorenzo Lizalde, J.I. (ed.). CDL de Aragón, Zaragoza: 19-26.

Aparicio, P. y Figueiredo, C. (2016): El grado de evidencia histórico-arqueológica de las reconstrucciones virtuales: Hacia una escala de representación gráfica. Revista Otarq. Otras arqueologías, 1: 235-247. DOI: http://dx.doi.org/10.23914/otarq.v0i1.96

Aranburu-Mendizabal, A., Camarero, C., García-Rojas, M., Gómez-Díez, F., González-García, A., Hernández, H.H., Perez-Arzak, U., Jindriska Pérez-Fernández, A.,

Alejandro Prieto, A. y Sigari, D. (2019): Una nueva ventana al conocimiento de las condiciones laborales de la arqueología en la España del año 2019. Revista Arkeogazte, 9: 39-63.

Armada, X-L. (2009): Indicadores bibliométricos, visibilidad y calidad de revistas científicas: en torno a Revista d'Arqueologia de Ponent. Revista d'Arqueologia de Ponent, 19: 7-28.

Armada, X.L. (2016): Explorando el panorama actual de las publicaciones periódicas de arqueología. Revista d'Arqueologia de Ponent en contexto. Revista d'Arqueologia de Ponent, 26: 295-310. DOI: https://doi.org/10.21001/rap.2016.26.19

Barreiro, D. y Varela-Pousa, R. (2017): La nueva ley de patrimonio cultural de Galicia: una lectura crítica. Nailos, 4: 163-191.

Belford, P. (2020): Ensuring Archaeology in the Planning System Delivers Public Benefit. Public Archaeology. DOI: https://doi.org/10.1080/14655187.2020.1833525

Benítez de Lugo, L. (2013): Régimen jurídico de la protección del Patrimonio Arqueológico en la Ley 4/2013 de Patrimonio Cultural de Castilla-La Mancha. Patrimonio Cultural y Derecho, 17: 263-278.

Bouso, M., Rafel, N. y Alonso, N. (eds.) (2016): Les revistes científiques d'arqueologia a debat: present i futur. Revista d'Arqueologia de Ponent, 26: 261-322.

Burgos Barrantes, B. (ed.) (2020): De lo público al bien común: emergencia de otros modelos de gestión del patrimonio cultural. Revista PH, 101. DOI: https://doi.org/10.33349/2020.101

Canosa Betés, J. y Díaz de Liaño, G. (2020): La carrera investigadora en arqueología y su impacto en la salud mental de los investigadores predoctorales. Complutum, 31(2): 379-401.

Castillo, A. (2004): La gestión del patrimonio arqueológico y el urbanismo en la Comunidad de Madrid. Complutum, 15: 99-144.

Castillo Mena, A. y Almansa Sánchez, J. (2021): Applied Research Through Community Building during International Conferences. The Case of the International Conference on Best Practices in World Heritage. Minorca, Spain. Journal of Community Archaeology and Heritage, 8(3): 192-209. DOI: https://doi.org /10.1080/20518196.2021.1894784

CCOO (2021): La modificación de la Ley de Ciencia 14/2011 condena al personal de la investigación a la precariedad estructural. URL: https://sae.fsc.ccoo.es/d1a922593b50c4612696c362e300249b000001. pdf [visitado 31/03/21]

CCOO[P] (2021): ANECA desmiente a los rectores: ¡Ya puede pedir Sexenios el PDI temporal! Blog de CCOO[P]recarios/as IDI. URL: https://ccoo-precarios.blogspot.com/2021/02/aneca-desmiente-losrectores-ya-puede.html (Último acceso, 31/03/21)

Cebrián, R. (2016): La gestión del patrimonio arqueológico en Castilla-La Mancha: La red de Parques Arqueológicos. Boletín “Arqueología somos todos”, 4: 13-15.

Colegio de Profesionales de la Arqueología (2020): Informe del impacto económico de la covid-19 en la arqueología profesional a nivel estatal. Colegio de Profesionales de la Arqueología del CDL de Madrid y 
Plataforma Estatal de Profesionales de la Arqueología, Madrid. URL: https://www.arqueologiademadridcdl.org/wp-content/uploads/2020/05/informe_encuesta_covid19.pdf [visitado 31/03/2021]

Comendador Rey, B. (2018): Arqueología pública en las aulas universitarias: Un primer balance de su implantación. Cuadernos de Prehistoria y Arqueología de la Universidad de Granada, 28: 9-24. DOI: https://doi.org/10.30827/cpag.v28i0.8455

Comisión Feminista Arkeogazte (2018): Protocolo de Arkeogazte para la prevención y actuación ante situaciones de acoso, abusos y agresiones sexuales o por razón de sexo. Revista Arkeogazte, 8: 35-46.

Corpas, N. (2020): Alternativas en resolución de conflictos en patrimonio arqueológico. El caso del expolio y el tráfico ilícito internacional. Revista d'Arqueologia de Ponent, 30: 337-348. DOI: https://doi. org/10.21001/rap.2020.30.18

Corpas, N. y Castillo, A. (2019): Tourism 3.0 and archaeology: approaching tourists' generated-content of World Heritage sites. Pasos. Revista de Turismo y Patrimonio Cultural, 17(1): 39-52. DOI: https://doi. org/10.25145/j.pasos.2019.17.003

Coto-Sarmiento, M., Delgado Anés, L., López Martínez, L., Martín Alonso, J., Pastor Pérez, A., Ruiz, A. y Yubero, M. (2020): Informe sobre el acoso sexual en arqueología (España). Zenodo, Barcelona, Granada y Madrid. DOI: https://doi.org/10.5281/zenodo.3662763

Criado Boado, F. (2011): El futuro de la arqueología española. En Almansa Sánchez 2011: 55-60.

Criado Boado, F., Alonso-Pablos, D., Blanco, M.J., Porto, Y., Rodríguez-Paz, A., Cabrejas, E., del BarrioÁlvarez, E. y Martínez, L.M. (2019): Coevolution of visual behaviour, the material world and social complexity, depicted by the eye-tracking of archaeological objects in humans. Scientific Reports, 9: 3985. DOI: https://doi.org/10.1038/s41598-019-39661-w

Demoule, J.P. (2016): Preventive Archaeology: Scientific Research or Commercial Activity? En Novaković et al. 2016: 9-20.

Díaz, D., Torija, A. y Zarco, E. (2014): Miles de anteayeres cargados de memoria... un futuro incierto. Apuntes de Arqueología. Boletín del CDL de Madrid, 246: 13-19.

Díaz de Liaño, G., Corpas, N. y Moral, E. (2020): Epílogo. (No) Todo son risas y diversión: el sufrimiento durante el trabajo de campo arqueológico. En García, J. 2011. Amor Estratigráfico. El Libro. JAS Arqueología Editorial, Madrid: 255-267.

Díaz Santiago, M.J. (2013): Experiencia no académica: el profesorado asociado como actor doblemente precarizado. Sociología del Trabajo, 78: 51-71.

Everill, P., Bennett, R. y Burnell, K. (2020): Dig in: An evaluation of the role of archaeological fieldwork for the improved wellbeing of military veterans. Antiquity, 94(373): 212-227. DOI: https://doi. org/10.15184/aqy.2019.85

Farrell-Banks, D. (2018): Can archaeology become a positive working environment for adults with autism spectrum disorders? Journal of Community Archaeology and Heritage, 5(3): 198-2010. DOI: https:// doi.org/10.1080/20518196.2018.1477038

Flexner, J. (2020): Degrowth and a sustainable future for archaeology. Archaeological dialogues, 27: 159171. DOI: https://doi.org/10.1017/S1380203820000203

García Fernández, J. (2014): La Ley 3/2013, de 18 de junio, de patrimonio histórico de la comunidad de Madrid, una norma redactada para facilitar la expoliación de bienes culturales. Apuntes de Arqueología. Boletín del CDL de Madrid, 246: 25-28.

Gibaja, J.F, Remolins, G., Valenzuela, S., Higuera, S., Nieto, A., Palma, O., González, A., Oms, X., Guzmán, V., Cubas, M., Masclans, A. y Mozota, M. (2021). Inclusive archaeology. Scientific outreach among 'forgotten collectives' in the streets of Barcelona (Spain). Journal of Community Archaeology and Heritage, 8(3): 160-177. DOI: https://doi.org/10.1080/20518196.2021.1873539

González Ruibal, A. (2008): Time to destroy: An archaeology of supermodernity. Current Anthropology, 49(2): 247-279. DOI: https://doi.org/10.1086/526099

González Ruibal, A. (2011): El desastre académico de la arqueología. En, Almansa Sánchez 2011: 99-104. González Ruibal, A. (2018): An Archaeology of the Contemporary Era. Routledge, London.

González Sánchez, C. (coord.) (2021): Comunicación y redes sociales en instituciones culturales. Revista PH, 102: 118-205. DOI: https://doi.org/10.33349/2021.102

Hernando, C. y Tejerizo, C. (2011): La Arqueología y la Academia: del siglo XIX al "Plan Bolonia". Revista Arkeogazte, 1: 53-69.

Howery, Ch.L. (2020): Archaeology of Austerity: The effects of the economic crisis on heritage in Greece. Tesis Doctoral. Universidad de Stanford. 
Junyent, E. y López J.B. (eds.) (2019): El finançament públic de la recerca aruqeològica i paleontológica a Catalunya. Projectes quadriennals de recerca en matèria d'arqueologia i paleontología. Revista d'Arqueologia de Ponent, 29: 193-205.

Lobera Serrano, J. (2020): La percepción social del patrimonio cultural en la Comunidad de Madrid. DGPC Comunidad de Madrid, Madrid.

López García, J.P. (2019): MASAV (Museo Abierto Sierra de Ávila y Valle Amblés). Propuesta para la supervivencia de los paisajes culturales de la provincia de Ávila a partir de su patrimonio histórico y arqueológico. Investigar el pasado para entender el presente: Homenaje al profesor Carmelo Luis López. Volumen 2. En Luis López, C. (ed.). Diputación de Ávila e Institución Gran Duque de Alba, Ávila: 365-384.

López Martínez, L. y Martín Alonso, J. (2018): ¿Han ganado ellas? Arqueólogas vs Constructoras. Reflexiones y posibles vías para salvar la arqueología de los depredadores de Cultura. Congreso Nacional de Arqueología Profesional (Zaragoza 2017). En Lorenzo Lizalde, J.I. (ed.). CDL de Aragón, Zaragoza: 225-233.

Martínez, B. y Querol, M.A. (1996): La gestión del Patrimonio Arqueológico en España. Alianza, Madrid.

MCU (2011): Encuesta de hábitos y prácticas culturales en España 2010-2011. Ministerio de Cultura, Madrid.

MCU (2019): Encuesta de hábitos y prácticas culturales en España 2018-2019. Ministerio de Cultura, Madrid.

Moral, E. (2016): Qu(e)erying sex and gender in archaeology: a critique of the "third" and other sexual categories. Journal of Archaeological Method and Theory 23(3): 788-809. DOI: https://doi.org/10.1007/ s10816-016-9294-y

Morín de Pablos, J. y Barroso Cabrera, R. (2014): De la arqueología de la mortadela a la arqueología de la resistencia. La democratización fallida de la Arqueología española. Arqueoweb, 15: 315-321.

Novaković, P., Horňák, M., Guermandi, M.P., Stäuble, H., Depaepe, P. y Demoule, J.P. (eds.) (2016): Recent Developments in Preventive Archaeology in Europe. Ljublana University Press, Ljublana. DOI: https://doi.org/10.4312/9789612378882

O'Mahony, T. (2015): Enabled Archaeology. Guide 41. BAJR Series. URL: http://www.bajr.org/ BAJRGuides/41_Enabled_Archaeology/41EnabledArchaeology.pdf [visitado 31/03/21]

Olivier, L. (2016): How I learned the Law of the Market. En Aparicio 2016: 223-238.

Parga-Dans, E., Barreiro, D. y Varela-Pousa, R. (2016): Isomorphism and legitimacy in Spanish contract archaeology: the free-fall of an institutional model and the caveat of change. International Journal of Heritage Studies, 22(4): 291-301. DOI: https://doi.org/10.1080/13527258.2015.1137621

Pérez Santos, E. (2020): Investigaciones de públicos en los museos: evolución histórica, problemas actuales y retos futuros. Dykinson, Madrid.

Plantzos, D. (2018): Crisis, austerity measures and beyond: archaeology in Greece since the global financial crisis. Archaeological Reports, 64: 171-180. DOI: https://doi.org/10.1017/S0570608418000261

Poulios, I. y Touloupa, S. (2018): Museums and Crisis: The Imperative to Achieve Strategic Agility in the Current Instability. A Case Study of the Major Archaeological Museums in Greece. Public Archaeology, 17(1): 3-35. DOI: https://doi.org/10.1080/14655187.2018.1553127

Querol, M.A. (2010): Manual de Gestión del Patrimonio Cultural. Akal, Madrid.

Querol, M.A. y Martínez Navarrete, M.I. (1994): La actividad arqueológica en España. Una delimitación pendiente. En 6 Coloquio Hispano-Ruso de Historia. Fundación Cultural Banesto, Madrid, 184-92.

Rodríguez Temiño, I. y Afonso Marrero, J.A. (2019): El necesario cambio de modelo en la Arqueología profesional española. Complutum, 30(1): 43-57. DOI: http://dx.doi.org/10.5209/CMPL.64507

Rodríguez Temiño, I., Yáñez, A., Jorge-Villar, S., Reyes Mateo, A., Rufino Rus, J., Salas Álvarez, J. y Lavín Berdonces, A.C. (2018): Forensic Archaeometry Applied to Antiquities Trafficking: The Beginnings of an Investigation at the Frontiers of Knowledge. Arts, 7(4): 98. DOI: https://doi.org/10.3390/arts7040098

Ruiz García, D. (2020): La socialización patrimonial a través de la recreación histórica y la arqueología experimental. Revista PH, 101: 284-297. DOI: https://doi.org/10.33349/2020.101.4637

Ruiz Zapatero, G. (2016): Publicar revistas de arqueología: cartografía actual y retos de futuro. Revista d'Arqueologia de Ponent, 26: 265-279. DOI: https://doi.org/10.21001/rap.2016.26.15

Sayer, F. (2015): Can digging make you happy? Archaeological excavations, happiness and heritage. Arts and Health, 7(3): 247-260. DOI: https://doi.org/10.1080/17533015.2015.1060615

Society of Antiquaries of London (2020): The Future of Archaeology in England. SAL, London. 
Tarlow, S. y Nilsson Stutz, L. (2013): Can an archaeology be a public intellectual? Archaeological Dialogues, 20(1): 1-5 + respuestas. DOI: https://doi.org/10.1017/S1380203813000032

Trow, S. (2016): 25 Years of Development-led Archaeology in England. En Novaković et al. 2016: 55-68.

Universidad Complutense de Madrid (2019): Informe de la encuesta a estudiantes, Curso 2018/2019. Grado de Arqueología. Vicerrectorado de Calidad UCM, Madrid. URL: https://geografiaehistoria.ucm. es/encuestas-de-satisfaccion-arqueologia [visitado 31/03/21]

Universidad de Granada (2018): Informes de satisfacción con la Titulación. Grado de Arqueología. Unidad de Calidad, Innovación Docente y Prospectiva URG, Granada. URL: https://grados.ugr.es/arqueologia/ static/CMSRemoteManagement/*/vic_cal/_dir_remotos/base_grado/_list_/indicadores?login_form register [visitado 31/03/21]

Watson, S. (2021): Archaeology and public Benefit. EAC symposium proceedings. Internet Archaeology, 57. DOI: https://doi.org/10.11141/ia.57.19

Wills, J. (2018): The world after PPG16: 21st-century challenges for archaeology. CIfA for Historic England. Reading.

Winterton, S. (2014): From the Army Medical Centre to Operation Nightingale: My entry into Archaeology. Journal of Community Archaeology and Heritage, 1(3): 245-247. DOI: https://doi.org/10.1179/205181 9614Z.00000000015

Zarzuela, P., Martín, J. y Donat, M. (2019): Una radiografía necesaria del sector desde una mirada de género. Arqueoweb, 19: 34-49.

Zorzin, N. (2015): Dystopian Archaeologies: the Implementation of the Logic of Capital in Heritage Management. International Journal of Historical Archaeology, 19: 791-809.

Zorzin, N. (2016): New managerial strategies in British commercial archaeology. En Aparicio 2016: 297326.

Zorzin, N. (2021): Is archaeology conceivable within the degrowth movement? Archaeological Dialogues, 28: 1-16. DOI: https://doi.org/10.1017/S1380203821000015 
